\title{
A New Optimized Adaptive Approach for Estimation of the Wigner Kernel
}

\author{
Venelin Todorov \\ Bulgarian Academy of Sciences \\ Institute of Mathematics and Informatics \\ ul. G. Bonchev 8, 1113 Sofia, Bulgaria \\ Bulgarian Academy of Sciences \\ Institute of Information and Communication Technologies \\ ul. G. Bonchev 25A, 1113 Sofia, Bulgaria \\ Email: vtodorov@math.bas.bg,venelin@parallel.bas.bg

\section{Ivan Dimov \\ Bulgarian Academy of Sciences} \\ Institute of Information and Communication Technologies \\ ul. G. Bonchev 25A, 1113 Sofia, Bulgaria \\ Email: ivdimov@bas.bg
}

\author{
Stefka Fidanova \\ Bulgarian Academy of Sciences \\ Institute of Information and Communication Technologies \\ ul. G. Bonchev 25A, 1113 Sofia, Bulgaria \\ Email: stefka@parallel.bas.bg
}

\begin{abstract}
In this paper we study numerically an optimized Adaptive Monte Carlo algorithm for the Wigner kernel - an important problem in quantum mechanics represented by difficult multidimensional integrals. We will show the advantages of the optimized Adaptive MC algorithm and compare the results with the Adaptive approach from our previous work [4] and other stochastic approaches for computing the Wigner kernel in 3,6,9dimensional case. The 12-dimensional case will be considered for the first time. A comprehensive study and an analysis of the computational complexity of the optimized Adaptive MC algorithm under consideration has also been presented.
\end{abstract}

\section{INTRODUCTION}

The Monte Carlo (MC) methods are widely used in solving different multidimensional problems by performing realizations of random processes or random variables. One of the best known physicist Richard Feynman formulated the problem of finding an effective and fast algorithm with linear or polynomial computational complexity for computing multidimensional integrals that represent Wigner kernel [2]. More information about the signed particle formulation of a singlebody and many-body system can be found in [3]. So far the Wigner kernel is calculated with deterministic methods which suffer from the „,curse of dimensionality” and this means

Venelin Todorov is supported by the Bulgarian National Science Fund under Young Scientists Project KP-06-M32/2 - 17.12.2019 "Advanced Stochastic and Deterministic Approaches for Large-Scale Problems of Computational Mathematics" and by the National Scientific Program "Information and Communication Technologies for a Single Digital Market in Science, Education and Security (ICT in SES)", contract No DO1-205/23.11.2018, financed by the Ministry of Education and Science in Bulgaria. Stoyan Poryazov is supported by the joint research project Symbolic-Numerical Decision Methods for Algebraic Systems of Equations in Perspective Telecommunication Tasks of IMI-BAS, Bulgaria and JINR, Dubna, Russia. The work is also supported by the Bulgarian National Science Fund under Project DN 12/5-2017 "Efficient Stochastic Methods and Algorithms for Large-Scale Problems". computational times growing exponentially with the problem dimension. Meanwhile stochastic MC methods are not affected by the „,curse of dimensionality”.

\section{DESCRIPTION OF THE OPTIMIZED AdAPTIVE APPROACH}

Adaptive approach [1] is well known method for evaluation of multidimensional integrals, especially when the integrand function has peculiarities and peaks. Let $p_{j}$ and $I_{\Omega_{j}}$ are the following expressions: $p_{j}=\int_{\Omega_{j}} p(\mathbf{x}) \mathrm{d} \mathbf{x}$ and $I_{\Omega_{j}}=$ $\int_{\Omega_{j}} f(\mathbf{x}) p(\mathbf{x}) \mathrm{d} \mathbf{x}$. Consider now a random point $\xi^{(j)} \in$ $\Omega_{j}$ with a density function $p(\mathbf{x}) / p_{j}$. In this case $I_{\Omega_{j}}=$ $\mathbf{E}\left[\frac{p_{j}}{N} \sum_{i=1}^{N} f\left(\xi_{i}^{(j)}\right)\right]=\mathbf{E} \theta_{N}$. This adaptive algorithm gives an approximation with an error $\varepsilon \leq c N^{-1 / 2}$, where $c \leq$ $0.6745 \sigma(\theta)(\sigma(\theta)$ is the standard deviation).

The optimized adaptive algorithm has higher accuracy than the original Adaptive Monte Carlo algorithm as can be seen from the tables below. The increase of the constant for the initial number of taken subregions $M=4$ improves the relative error compared with the previous choice $M=2$ in [4]. The optimized adaptive algorithm is described below.

\section{Algorithm}

1. Input data: total number of points $N 1$, constant $M=4$ (the initial number of subregions taken), constant $\varepsilon$ (max value of the variance in each subregion), constant $\delta$ (maximal admissible number of subregions), d-dimensionality of the initial region/domain, $f$ - the function of interest.

1.1. Calculate the number of points to be taken in each subregion $N=N 1 / \delta$. 
2. $\quad$ For $j=1, M^{d}$ :

2.1. Calculate the approximation of $I_{\Omega_{j}}$ and the variance $\mathbf{D}_{\Omega_{j}}$ in subdomain $\Omega_{j}$ based on $N$ independent realizations of random variable $\theta_{N}$;

2.2. If $\left(\mathbf{D}_{\Omega_{j}} \geq \varepsilon\right)$ then

2.2.1. Choose the axis direction on which the partition will perform,

2.2.2. Divide the current domain into two $\left(G_{j_{1}}, G_{j_{2}}\right)$ along the chosen direction,

2.2.3. If the length of obtained subinterval is less than $\delta$ then go to step 2.2.1 else $j=j_{1} G_{j_{1}}$ is the current domain right and go to step 2.1;

2.3. Else if $\left(\mathbf{D}_{\Omega_{j}}<\varepsilon\right)$ but an approximation of $I_{G_{j_{2}}}$ has not been calculated yet, then $j=j_{2} G_{j_{2}}$ is the current domain along the corresponding direction right and go to step 2.1;

2.4. Else if $\left(\mathbf{D}_{\Omega_{j}}<\varepsilon\right)$ but there are subdomains along the other axis directions, then go to step 2.1;

2.5. Else Accumulation in the approximation $I_{N}$ of $I$.

For the simple case when we have the two dimensional case $(N=2)$ and on the first step in the optimized adaptive approach we have $M=4$ subdomains in our optimized Adaptive approach and

$$
\hat{\theta}_{N}=\frac{1}{N_{1}} \sum_{i=1}^{N_{1}} \theta_{i}+\frac{1}{N_{2}} \sum_{i=1}^{N_{2}} \theta_{i}+\frac{1}{N_{3}} \sum_{i=1}^{N_{3}} \theta_{i}+\frac{1}{N_{4}} \sum_{i=1}^{N_{4}} \theta_{i},
$$

where $N_{1}+N_{2}+N_{3}+N_{4}=N$, so we have the same number of operations as the Crude Monte Carlo, which computational complexity is linear, to evaluate an approximation of $I_{G_{j}}$.

So we choose only $\mathcal{O}(1)$ subdomains where the variance is greater than the parameter $\varepsilon$ and this is independent of $N$. When we divide the domain on every step adaptiveness is not in all subdomains, but only in $\mathcal{O}(1)$ subdomains. At the beginning we have to choose $\frac{N}{k_{0}}$ random points. After that when dividing the domain into $2^{N}$ subdomains, we choose only $\mathcal{O}(1)$ subdomains, this choice is again independent of $N$. In these subdomains we choose $\frac{N}{k_{1}}$ points. On the $j^{t h}$ step of the Adaptive approach we choose $\mathcal{O}(1)$ subdomains with $\frac{N}{k_{j}}$ points. We have that $\sum_{j=0}^{i} \frac{1}{k_{j}}=1$. Therefore for the computational complexity we obtain

$$
\begin{gathered}
\frac{N}{k_{0}}+\mathcal{O}(1) \frac{N}{k_{1}}+\cdots+\mathcal{O}(1) \frac{N}{k_{i}}= \\
=N \mathcal{O}(1)\left(\sum_{j=0}^{i} \frac{1}{k_{j}}\right)=N \mathcal{O}(1)=\mathcal{O}(N) .
\end{gathered}
$$

In this way we can conclude that the computational complexity of the optimized Adaptive algorithm is linear.

\section{NUMERICAL EXAMPLES}

A new formulation of quantum mechanics in terms of signed classical field-less particles is presented in [3]. Just for completeness we give here the three postulates which completely define the new mathematical formulation of quantum mechanics taken from [3].

Postulate I. Physical systems can be described by means of (virtual) Newtonian particles, i.e. provided with a position $\mathrm{x}$ and a momentum $\mathbf{p}$ simultaneously, which carry a sign which can be positive or negative.

Postulate II. A signed particle, evolving in a potential $V=V(x)$, behaves as a field-less classical point-particle which, during the time interval $\mathrm{d} t$, creates a new pair of signed particles with a probability $\gamma(\mathbf{x}(t)) \mathrm{d} t$, where

$\gamma(\mathbf{x})=\int_{-\infty}^{+\infty} \operatorname{D} \mathbf{p}^{\prime} V_{W}^{+}\left(\mathbf{x} ; \mathbf{p}^{\prime}\right) \equiv \lim _{\triangle \mathbf{p}^{\prime} \rightarrow 0^{+}} \sum_{\mathbf{M}=-\infty}^{+\infty} V_{W}^{+}\left(\mathbf{x} ; \mathbf{M} \triangle \mathbf{p}^{\prime}\right)$,

where $\hbar=\frac{h}{2 \pi}$ is the reduced Planck constant $(h)$ or Dirac constant, $\mathbf{M}=\left(M_{1}, M_{2}, \ldots, M_{d}\right)$ is a set of $d$ integers and $V_{W}^{+}(\mathbf{x} ; \mathbf{p})$ is the positive part of the quantity

$V_{W}(\mathbf{x} ; \mathbf{p})=\frac{i}{\pi^{d} \hbar^{d+1}} \int_{-\infty}^{+\infty} \mathrm{d} \mathbf{x}^{\prime} e^{-\frac{2 i}{\hbar} \mathbf{x}^{\prime}} \mathbf{p}\left[V\left(\mathbf{x}+\mathbf{x}^{\prime}\right)-V\left(\mathbf{x}-\mathbf{x}^{\prime}\right)\right]$,

known as the Wigner kernel (in a d-dimensional space) [5]. If, at the moment of creation, the parent particle has sign $s$, position $\mathbf{x}$ and momentum $\mathbf{p}$, the new particles are both located in $\mathbf{x}$, have signs $+s$ and $-s$, and momentum $\mathbf{p}+\mathbf{p}^{\prime}$ and $\mathbf{p}-\mathbf{p}^{\prime}$ respectively, with $\mathbf{p}^{\prime}$ chosen randomly according to the (normalized) probability $\frac{V_{W}^{+}(\mathbf{x} ; \mathbf{p})}{\gamma(\mathbf{x})}$.

Postulate III. Two particles with opposite sign and same phase-space coordinates $(\mathbf{x}, \mathbf{p})$ annihilate.

The infinite domain of integration can be mapped into the $s$ dimensional unit hypercube using the following transformation $\frac{1}{2}+\frac{1}{\pi} \arctan (x)$ which maps $(-\infty, \infty)$ to $(0,1)$. We want to compute (1) in the $3,6,9$ and for the first time in 12 dimensional case,

$$
V w(x, p)=\int e^{\left(\frac{-i 2 \sum_{k=1}^{n} x_{k}^{\prime} p_{k}}{\hbar}\right)} \times
$$

$\left[V\left(x_{1}+x_{1}^{\prime}, \ldots x_{n}+x_{n}^{\prime}\right)-V\left(x_{1}-x_{1}^{\prime}, \ldots x_{n}-x_{n}^{\prime}\right)\right] d x_{1}^{\prime} \ldots d x_{n}^{\prime}$,

where the Wigner potential is $V=V(x)=$ $\left\{x_{1} \ldots x_{n}, x^{\prime}, x, p, x+x^{\prime}, x-x^{\prime} \in[0,1]\right\}$. It is well known that Wigner kernel has real values [5].

First, we will make a comparison with deterministic method of mid rectangulars, and after that with the well known stochastic approaches of Sobol QMC and Fibonacci based lattice rule FIBO, see [4].

In Table I it can be seen that the optimized stochastic approach gives better results and lower relative errors than the adaptive approach used in our previous study [4]. It can be 
Table I

RELATIVE ERROR OF THE OPTIMIZED ADAPTIVE APPROACH, ADAPTIVE APPROACH AND THE DETERMINISTIC MID RECTANGULAR METHOD

\begin{tabular}{|c|c|c|c|c|c|c|c|}
\hline \hline $\mathrm{s}$ & $\mathrm{N}$ & determ. & $\mathrm{t}(\mathrm{s})$ & OptAdapt & $\mathrm{t}(\mathrm{s})$ & Adapt & $\mathrm{t}(\mathrm{s})$ \\
\hline \hline \multirow{4}{*}{3} & $32^{2} \times 50$ & $8.51 \mathrm{e}-03$ & 0.2 & $1.47 \mathrm{e}-03$ & 0.1 & $2.71 \mathrm{e}-03$ & 0.1 \\
\cline { 2 - 8 } & $32^{2} \times 100$ & $8.21 \mathrm{e}-03$ & 0.5 & $1.14 \mathrm{e}-04$ & 0.21 & $3.42 \mathrm{e}-04$ & 0.2 \\
\cline { 2 - 8 } & $64^{2} \times 50$ & $5.76 \mathrm{e}-03$ & 1 & $5.12 \mathrm{e}-0$ & 0.6 & $7.52 \mathrm{e}-05$ & 0.55 \\
\cline { 2 - 8 } & $64^{2} \times 100$ & $4.89 \mathrm{e}-03$ & 1.9 & $7.11 \mathrm{e}-06$ & 1.4 & $1.21 \mathrm{e}-05$ & 1.3 \\
\hline \hline \multirow{4}{*}{6} & $8^{4} \times 50^{2}$ & $1.16 \mathrm{e}-02$ & 41.2 & $8.64 \mathrm{e}-05$ & 19.5 & $9.09 \mathrm{e}-04$ & 18.1 \\
\cline { 2 - 8 } & $8^{4} \times 100^{2}$ & $9.75 \mathrm{e}-03$ & 160.6 & $5.21 \mathrm{e}-06$ & 59.4 & $1.52 \mathrm{e}-05$ & 57.9 \\
\cline { 2 - 8 } & $16^{4} \times 50^{2}$ & $7.84 \mathrm{e}-03$ & 635.2 & $3.21 \mathrm{e}-05$ & 321 & $4.37 \mathrm{e}-04$ & 311.5 \\
\cline { 2 - 8 } & $16^{4} \times 100^{2}$ & $2.12 \mathrm{e}-03$ & 2469.1 & $2.13 \mathrm{e}-05$ & 1001.6 & $3.80 \mathrm{e}-04$ & 987.1 \\
\hline \hline \multirow{4}{*}{9} & $6^{6} \times 16^{3}$ & $1.75 \mathrm{e}-03$ & 835.5 & $5.11 \mathrm{e}-05$ & 345 & $7.62 \mathrm{e}-05$ & 330.5 \\
\cline { 2 - 8 } 9 & $6^{6} \times 32^{3}$ & $1.35 \mathrm{e}-03$ & 5544.1 & $1.41 \mathrm{e}-05$ & 2133.6 & $2.73 \mathrm{e}-05$ & 2225.1 \\
\cline { 2 - 8 } & $6^{6} \times 40^{3}$ & $1.12 \mathrm{e}-03$ & 10684.4 & $1.67 \mathrm{e}-06$ & 4531.5 & $8.12 \mathrm{e}-06$ & 4491.5 \\
\hline
\end{tabular}
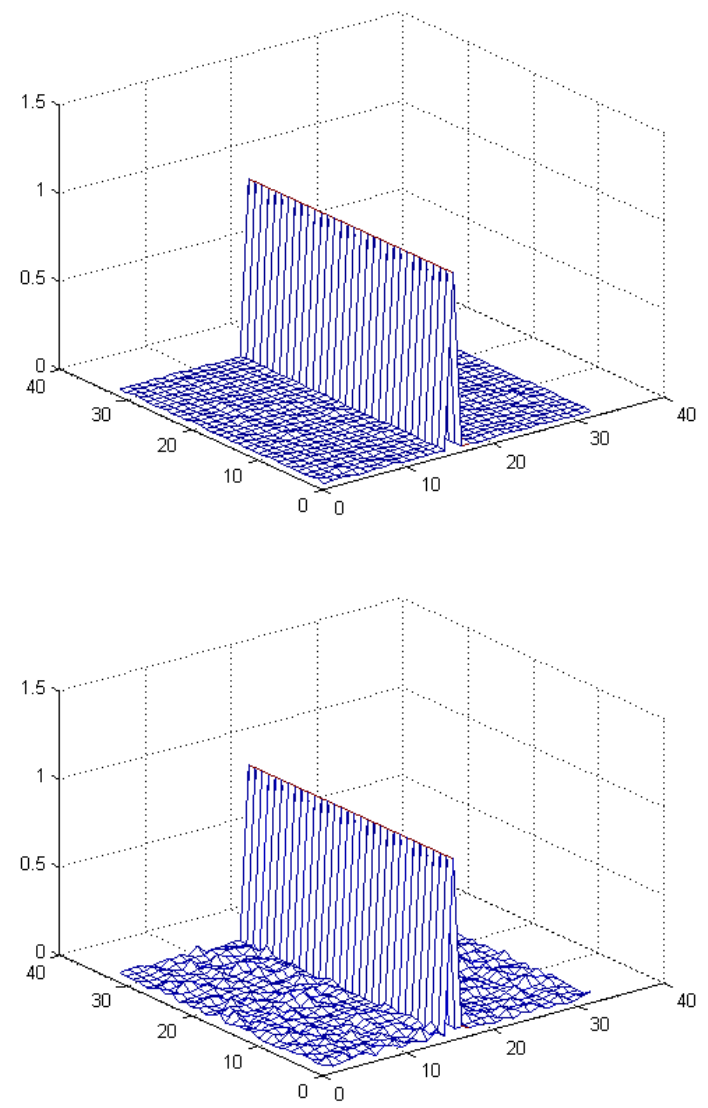

Figure 1. The Wigner kernel with optimized adaptive and standard adaptive method

seen that the computational time for the optimized Adaptive MC approach is better than the deterministic method when the dimensionality increases. The advantage of the optimized
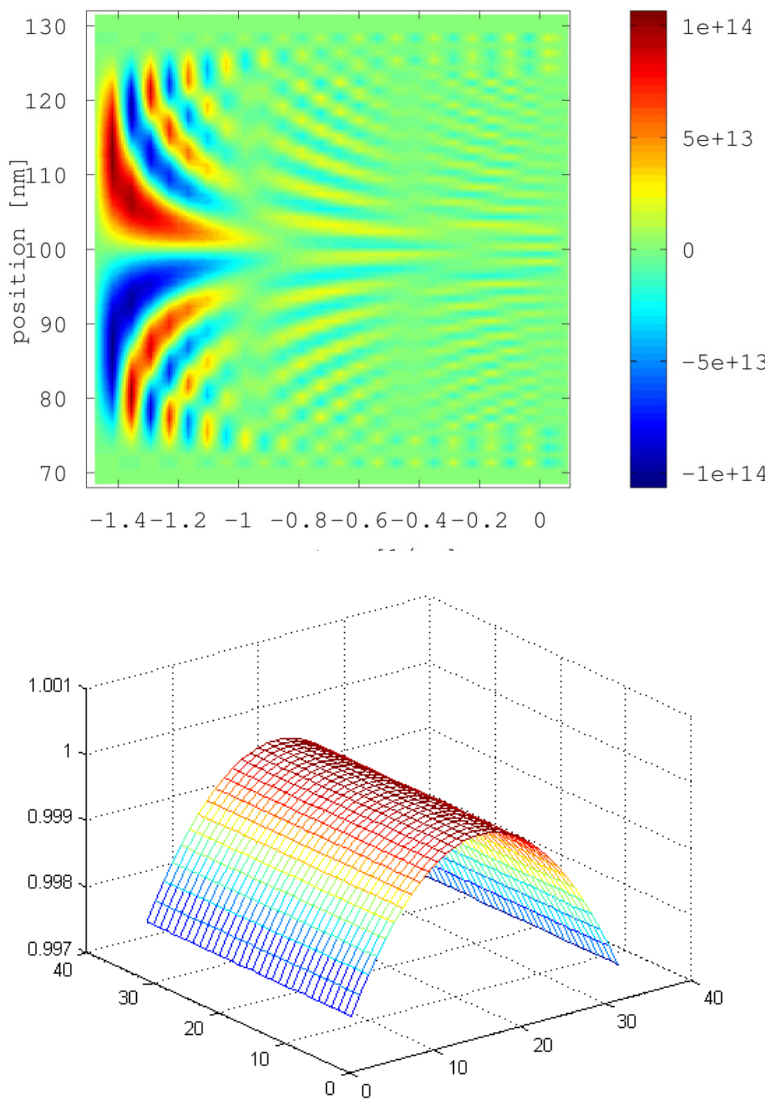

Figure 2. The position and the peak of the Wigner kernel with optimizes adaptive approach

Table II

RELATIVE ERROR FOR 3 DIMENSION

\begin{tabular}{|c|c|c|c|c|c|c|c|c|}
\hline \hline $\mathrm{N}$ & Adapt & t,s & OptAdapt & t,s & FIBO & t,s & Sobol & t,s \\
\hline $10^{3}$ & $5.36 \mathrm{e}-03$ & 0.3 & $6.75 \mathrm{e}-04$ & 0.4 & $3.72 \mathrm{e}-02$ & 0.02 & $1.07 \mathrm{e}-02$ & 0.05 \\
\hline $10^{4}$ & $4.84 \mathrm{e}-04$ & 2.9 & $8.15 \mathrm{e}-05$ & 3.3 & $7.06 \mathrm{e}-03$ & 0.07 & $8.77 \mathrm{e}-03$ & 0.54 \\
\hline $10^{5}$ & $2.51 \mathrm{e}-05$ & 29 & $5.01 \mathrm{e}-06$ & 32.6 & $3.40 \mathrm{e}-03$ & 0.43 & $8.57 \mathrm{e}-04$ & 5.74 \\
\hline $10^{6}$ & $1.76 \mathrm{e}-05$ & 287 & $4.38 \mathrm{e}-07$ & 302 & $1.01 \mathrm{e}-03$ & 4.4 & $6.73 \mathrm{e}-04$ & 51.6 \\
\hline $10^{7}$ & $6.26 \mathrm{e}-06$ & 2535 & $8.02 \mathrm{e}-08$ & 2708 & $1.80 \mathrm{e}-04$ & 49.7 & $5.98 \mathrm{e}-05$ & 499 \\
\hline
\end{tabular}

Table III

RELATIVE ERROR FOR 3 DIMENSION

\begin{tabular}{|c|c|c|c|c|}
\hline \hline $\mathrm{s}$ & Adapt & OptAdapt & FIBO & Sobol \\
\hline 0.1 & $6.74 \mathrm{e}-03$ & $8.73 \mathrm{e}-04$ & $8.12 \mathrm{e}-03$ & $1.01 \mathrm{e}-02$ \\
\hline 1 & $8.73 \mathrm{e}-04$ & $4.05 \mathrm{e}-05$ & $5.42 \mathrm{e}-03$ & $7.27 \mathrm{e}-03$ \\
\hline 10 & $5.62 \mathrm{e}-05$ & $9.12 \mathrm{e}-06$ & $2.11 \mathrm{e}-03$ & $7.83 \mathrm{e}-04$ \\
\hline 100 & $3.43 \mathrm{e}-06$ & $8.18 \mathrm{e}-07$ & $9.50 \mathrm{e}-04$ & $2.18 \mathrm{e}-04$ \\
\hline
\end{tabular}

adaptive algorithm in comparison with the previously used adaptive algorithm is shown on Figure 1, and the computation of the position of the signs and the peak are given in Figure 2. The numerical results including relative errors and 
Table IV

RELATIVE ERROR FOR 6 DIMENSION
Table IX

RELATIVE ERROR FOR 12 DIMENSION

\begin{tabular}{|c|c|c|c|c|c|c|c|c|}
\hline \hline $\mathrm{N}$ & Adapt & t,s & OptAdapt & t,s & FIBO & t,s & Sobol & t,s \\
\hline $10^{3}$ & $6.72 \mathrm{e}-03$ & 0.41 & $2.23 \mathrm{e}-04$ & 0.5 & $7.82 \mathrm{e}-03$ & 0.01 & $2.42 \mathrm{e}-02$ & 0.09 \\
\hline $10^{4}$ & $9.10 \mathrm{e}-04$ & 3.5 & $4.74 \mathrm{e}-05$ & 4.1 & $5.01 \mathrm{e}-03$ & 0.07 & $5.02 \mathrm{e}-03$ & 0.78 \\
\hline $10^{5}$ & $5.26 \mathrm{e}-05$ & 33 & $5.43 \mathrm{e}-06$ & 37 & $6.88 \mathrm{e}-03$ & 0.43 & $4.60 \mathrm{e}-04$ & 7.19 \\
\hline $10^{6}$ & $2.70 \mathrm{e}-06$ & 315 & $5.04 \mathrm{e}-07$ & 351 & $7.68 \mathrm{e}-04$ & 5.97 & $3.59 \mathrm{e}-04$ & 73 \\
\hline $10^{7}$ & $1.03 \mathrm{e}-06$ & 2438 & $8.12 \mathrm{e}-08$ & 2841 & $4.12 \mathrm{e}-04$ & 48 & $8.11 \mathrm{e}-05$ & 590 \\
\hline
\end{tabular}

Table V

RELATIVE ERROR FOR 6 DIMENSION

\begin{tabular}{|c|c|c|c|c|}
\hline \hline t,s & Adapt & OptAdapt & FIBO & Sobol \\
\hline 0.1 & $9.25 \mathrm{e}-04$ & $6.81 \mathrm{e}-04$ & $8.11 \mathrm{e}-03$ & $2.13 \mathrm{e}-02$ \\
\hline 1 & $4.51 \mathrm{e}-04$ & $9.09 \mathrm{e}-05$ & $9.25 \mathrm{e}-04$ & $3.31 \mathrm{e}-03$ \\
\hline 10 & $2.57 \mathrm{e}-05$ & $8.13 \mathrm{e}-06$ & $5.11 \mathrm{e}-04$ & $9.34 \mathrm{e}-04$ \\
\hline 100 & $2.72 \mathrm{e}-06$ & $5.08 \mathrm{e}-07$ & $1.05 \mathrm{e}-04$ & $1.27 \mathrm{e}-04$ \\
\hline
\end{tabular}

Table VI

RELATIVE ERROR FOR 9 DIMENSION

\begin{tabular}{|c|c|c|c|c|c|c|c|c|}
\hline \hline $\mathrm{N}$ & Adapt & t,s & OptAdapt & t,s & FIBO & t,s & Sobol & t,s \\
\hline $10^{3}$ & $4.92 \mathrm{e}-02$ & 0.4 & $8.23 \mathrm{e}-04$ & 0.5 & $2.03 \mathrm{e}-02$ & 0.06 & $5.42 \mathrm{e}-02$ & 0.11 \\
\hline $10^{4}$ & $9.09 \mathrm{e}-04$ & 3.9 & $2.02 \mathrm{e}-05$ & 4.7 & $2.02 \mathrm{e}-03$ & 0.07 & $6.02 \mathrm{e}-03$ & 0.88 \\
\hline $10^{5}$ & $3.32 \mathrm{e}-05$ & 35 & $1.08 \mathrm{e}-06$ & 40 & $9.16 \mathrm{e}-04$ & 0.53 & $3.57 \mathrm{e}-03$ & 7.56 \\
\hline $10^{6}$ & $6.46 \mathrm{e}-06$ & 367 & $4.14 \mathrm{e}-07$ & 381 & $7.13 \mathrm{e}-04$ & 3.7 & $8.02 \mathrm{e}-04$ & 72 \\
\hline $10^{7}$ & $1.21 \mathrm{e}-06$ & 2742 & $8.91 \mathrm{e}-08$ & 2912 & $4.84 \mathrm{e}-04$ & 40 & $5.19 \mathrm{e}-04$ & 621 \\
\hline
\end{tabular}

Table VII

RELATIVE ERROR FOR 9 DIMENSION

\begin{tabular}{|c|c|c|c|c|}
\hline \hline t,s & Adapt & OptAdapt & FIBO & Sobol \\
\hline 0.1 & $9.24 \mathrm{e}-03$ & $1.73 \mathrm{e}-03$ & $1.35 \mathrm{e}-03$ & $5.42 \mathrm{e}-02$ \\
\hline 1 & $1.23 \mathrm{e}-03$ & $8.05 \mathrm{e}-05$ & $8.72 \mathrm{e}-04$ & $5.59 \mathrm{e}-03$ \\
\hline 10 & $3.82 \mathrm{e}-05$ & $6.32 \mathrm{e}-06$ & $6.51 \mathrm{e}-04$ & $5.84 \mathrm{e}-03$ \\
\hline 100 & $3.09 \mathrm{e}-06$ & $7.58 \mathrm{e}-07$ & $3.70 \mathrm{e}-04$ & $6.39 \mathrm{e}-04$ \\
\hline
\end{tabular}

Table VIII

RELATIVE ERROR FOR 12 DIMENSION

\begin{tabular}{|c|c|c|c|c|c|c|c|c|}
\hline \hline $\mathrm{N}$ & Adapt & t,s & OptAdapt & $\mathrm{t}, \mathrm{s}$ & FIBO & t,s & Sobol & t,s \\
\hline $10^{3}$ & $3.91 \mathrm{e}-03$ & 0.7 & $3.21 \mathrm{e}-04$ & 0.9 & $1.33 \mathrm{e}-02$ & 0.09 & $2.85 \mathrm{e}-02$ & 0.2 \\
\hline $10^{4}$ & $5.04 \mathrm{e}-04$ & 4.5 & $1.08 \mathrm{e}-05$ & 6.1 & $1.34 \mathrm{e}-03$ & 0.1 & $4.04 \mathrm{e}-03$ & 1.34 \\
\hline $10^{5}$ & $2.76 \mathrm{e}-04$ & 48 & $5.04 \mathrm{e}-06$ & 56 & $5.51 \mathrm{e}-04$ & 0.68 & $1.77 \mathrm{e}-03$ & 9.8 \\
\hline $10^{6}$ & $4.14 \mathrm{e}-05$ & 415 & $2.72 \mathrm{e}-07$ & 432 & $4.43 \mathrm{e}-04$ & 4.7 & $4.07 \mathrm{e}-04$ & 82 \\
\hline $10^{7}$ & $2.31 \mathrm{e}-06$ & 3351 & $4.87 \mathrm{e}-08$ & 3467 & $2.5684 \mathrm{e}-04$ & 60 & $2.7 \mathrm{e}-04$ & 700 \\
\hline \hline
\end{tabular}

computational times corresponding to the algorithms under consideration are presented, and the algorithms efficiency is discussed. A numerical comparison for a given number of samples between the adaptive approach (Adapt) used in [4], the Sobol (Sob) and the Lattice sequences FIBO described in [4] and the new optimized Adaptive approach (OptAdapt) has been given in Tables II,IV,VI. From the all experiments

\begin{tabular}{|c|c|c|c|c|}
\hline \hline t,s & Adapt & OptAdapt & FIBO & Sobol \\
\hline 0.1 & $4.66 \mathrm{e}-03$ & $6.22 \mathrm{e}-04$ & $6.56 \mathrm{e}-04$ & $2.67 \mathrm{e}-02$ \\
\hline 1 & $3.25 \mathrm{e}-04$ & $4.51 \mathrm{e}-05$ & $4.45 \mathrm{e}-04$ & $2.98 \mathrm{e}-03$ \\
\hline 10 & $3.24 \mathrm{e}-05$ & $3.56 \mathrm{e}-06$ & $3.56 \mathrm{e}-04$ & $2.45 \mathrm{e}-03$ \\
\hline 100 & $1.31 \mathrm{e}-05$ & $4.16 \mathrm{e}-07$ & $1.87 \mathrm{e}-04$ & $3.21 \mathrm{e}-04$ \\
\hline
\end{tabular}

it can be clearly seen that the optimized adaptive approach gives relative errors with at least 1 or 2 orders better than those produced by the adaptive approach for the cost of slightly bigger computational times, because of the increased number of subregions taken in every subdomain $M$. The adaptive approach itself gives superior results to the other two stochastic approaches as it is completely described in our previous study [4]. The optimized adaptive MC algorithm is the slowest, but it requires smaller number of random points to achieve better accuracy even for higher dimensions and for a fixed computational time it gives the best relative error by at least 1 order, as can be seen from Tables III,V,VII. The optimized Adaptive MC approach outperforms the other two approaches FIBO and Sobol QMC by at least $3-5$ even for 12 dimensional case, see Table VIII,IX. The efficiency of the optimized adaptive MC algorithm is clearly shown in the case of Wigner kernel, where the integrand have computational specialty in the local subarea of the integration domain - see Figure 1 and how the peak is approximated by the optimized adaptive approach, see Figure 2.

\section{CONCLUSIONS}

The optimized adaptive Monte Carlo algorithm under consideration gives the most accurate results in computing the Wigner kernel by a stochastic approach and it has lower computational complexity than the existing deterministic approaches. This means that the proposed optimized stochastic approach is of great importance for the problems in quantum mechanics with high dimensions. Therefore, the presented optimized adaptive MC algorithm is one new successful solution (in terms of robustness and reliability) of Richard Feynman's problem for Wigner kernel evaluation.

\section{REFERENCES}

[1] Berntsen J., Espelid T.O., Genz A. (1991) An adaptive algorithm for the approximate calculation of multiple integrals, ACM Trans. Math. Softw. 17: 437-451, https://doi.org/10.1145/210232.210233.

[2] Feynman R.P. (1948) Space-time approach to nonrelativistic quantum mechanics, Rev. Mod. Phys. 20 , https://doi.org/10.1103/RevModPhys.20.367.

[3] Sellier J.M., Nedjalkov M., Dimov I. (2015) An introduction to applied quantum mechanics in the Wigner Monte Carlo formalism, Physics Reports Volume 577: 1-34, https://doi.org/10.1016/j.physrep.2015.03.001.

[4] Todorov, V., Dimov, I., Georgieva, R., \& Dimitrov, S. Adaptive Monte Carlo algorithm for Wigner kernel evaluation. Neural Comput \& Applic 32, 9953-9964 (2020). https://doi.org/10.1007/s00521-019-04519-9.

[5] Wigner E. (1932) On the quantum correction for thermodynamic equilibrium, Phys. Rev. 40: 749, https://doi.org/10.1103/PhysRev.40.749. 